\title{
Periprosthetic Joint Infection in Patients with Inflammatory Joint Disease: a Review of Risk Factors and Current Approaches to Diagnosis and Management
}

\author{
Todd A. Morrison, BS • Mark Figgie, MD • Andy O. Miller, MD • Susan M. Goodman, MD
}

Received: 22 November 2012/Accepted: 9 April 2013 /Published online: 3 July 2013

(C) Hospital for Special Surgery 2013

Original Release Date: June 15, 2013

Expiration Date: June 15, 2014

\section{Overview}

As the use of prosthetic joints increases, the incidence of prosthetic joint infection (PJI) is also increasing. Indications of inflammatory joint disease are easily missed, as these patients frequently have elevated inflammatory markers such as ESR and CRP, commonly used as screening tests for infection. Moreover, patients with inflammatory arthritis are at increased risk for PJI due to the use of immunosuppressant medications, as well as the immune dysfunction typical of inflammatory diseases. Given the importance of early diagnosis and treatment related to PJI, understanding the optimal management of potent immunosuppressants in the perioperative period is critical in avoiding early PJI.

\section{Learning Objectives}

Hospital for Special Surgery activities are intended to improve the quality of patient care and safety. At the conclusion of this course, the participant should be able to:

- Recognize the signs and symptoms of PJI, such as increase in pain and swelling, drainage from the joint, or systemic features such as fever or fatigue, in patients with inflammatory arthritis.

- Describe and safely prescribe DMARDs, such as methotrexate and plaquenil, to avoid increased risk of infection and achieve optimal outcomes.

- Implement additional work-up that may be necessary if certain inflammatory markers, such as ESR and CRP levels, are elevated in patients with PJI who have inflammatory arthritis.

- Understand the treatment options and the risks and benefits of two-stage revision or resection arthroplasty, compared to other therapies when PJI is diagnosed in patients with inflammatory arthritis.

$\overline{\text { Sponsored }}$ by Hospital for Special Surgery Office of Continuing Medical Education

\section{Target Audience}

This activity is targeted to Family Medicine, Fellows, General Medicine, Medical Students, Orthopaedic Surgeons, Residents, and Rheumatologists.

\section{Accreditation}

Hospital for Special Surgery is accredited by the Accreditation Council for Continuing Medical Education to provide continuing medical education for physicians.

\section{Credit Designation}

Hospital for Special Surgery designates this Journal-based activity for a maximum of 1.0 AMA PRA Category 1 Credit $(s)^{\mathrm{TM}}$. Physicians should claim only the credit commensurate with the extent of their participation in the activity.

\section{Commercial Support}

This journal-based activity did not receive commercial support.

\section{Faculty Disclosure:}

In accordance with the Accreditation Council for Continuing Medical Education's Standards for Commercial Support, all CME providers are required to disclose to the activity audience the relevant financial relationships of the planners, teachers, and authors involved in the development of CME content. An individual has a relevant financial relationship if he or she has a financial relationship in any amount occurring in the last 12 months with a commercial interest whose products or services are discussed in the CME activity content over which the individual has control.

It is the policy of Hospital for Special Surgery to disclose all financial relationships that planners, teachers, and authors have with commercial interests. 


\section{Activity Director Disclosure:}

Susan Goodman, MD has disclosed no financial relationships. Todd A. Morrison, BS has disclosed no financial relationships.

\section{Planning Committee Disclosure:}

Charles N. Cornell, MD has disclosed the following financial relationships:

Exactech Royalty

Merk Sharpe \& Dohme Corp. Expert Witness Testimony

Dr. Cornell will not be discussing products/services of the commercial interests with which relationships exist.

Tonie Costello, LCSW has disclosed no financial relationships.

Amy R. DeRobertis, MA has disclosed the following financial relationships:

Pfizer

Ownership Interest

Ms. DeRobertis will not be discussing products/services of the commercial interests with which relationships exist.

Mark P. Figgie, MD has disclosed the following financial relationships:

Mekanica

Ownership Interest

Ethicon

Supported/Contracted Funding

Dr. Figgie will not be discussing products/services of the commercial interests with which relationships exist.

Natanya Gayle, MPH has disclosed no financial relationships.

Allison Goldberg, MPA has disclosed no financial relationships.

Susan Goodman, MD has disclosed no financial relationships.

Andy O. Miller, MD has disclosed no financial relationships.

Todd A. Morrison, BS has disclosed no financial relationships.

\section{OCME/CME Committee Disclosure}

Hospital for Special Surgery Office of CME staff and CME Committee members have no relevant financial relationships to disclose regarding this activity.

\section{Activity Faculty}

Activity Directors:

Susan Goodman, MD

Assistant Attending Physician

Hospital for Special Surgery

Assistant Professor of Medicine

Weill Cornell Medical College

New York, NY
Todd A. Morrison, BS

Medical Student

Jefferson Medical College

Philadelphia, PA

Planning Committee:

Charles N. Cornell, MD

Clinical Director of Orthopaedic Surgery

Attending Orthopaedic Surgeon

Hospital for Special Surgery

New York, NY

Tonie Costello, LCSW

Program Coordinator, Accreditation and Compliance

Education \& Academic Affairs

Hospital for Special Surgery

New York, NY

Amy R. DeRobertis, MA

Associate Director, CME

Education \& Academic Affairs

Hospital for Special Surgery

New York, NY

Mark P. Figgie, MD

Chief of Surgical Arthritis Service

Attending Orthopaedic Surgeon

Hospital for Special Surgery

Professor of Clinical Orthopaedic Surgery

New York, NY

Natanya Gayle, MPH

Managing Editor, HSS Journal

Education \& Academic Affairs

Hospital for Special Surgery

New York, NY

Allison Goldberg, MPA

Director, Professional Education

Education \& Academic Affairs

Hospital for Special Surgery

New York, NY

Susan Goodman, MD

Assistant Attending Physician

Hospital for Special Surgery

Assistant Professor of Medicine

Weill Cornell Medical CollegeNew York, NY

Andy O. Miller, MD

Assistant Attending Physician

Hospital for Special Surgery

Assistant Professor of Clinical Medicine

Weill Cornell Medical College

New York, NY

Todd A. Morrison, BS

Medical Student

Jefferson Medical College

Philadelphia, PA 
For technical requirements related to this activity, please refer to the information below or contact Sonic Foundry.

\section{Windows}

- Microsoft Windows XP, Windows Server 2003, Windows Vista, Windows Server 2008, Windows 7 operating system

- Microsoft Internet Explorer 6.0 SP1 or later, Firefox 2.0 or later, or Google Chrome 1.0 (Chrome is only supported on Mediasite version 5.0.3 and later) Web browser

- Windows Media Player 9 or later

- For Firefox and Chrome playback, Microsoft Silverlight 1.0 or later (viewers are prompted to install this plug-in when attempting to view a presentation)

- Broadband internet connection (256 Kbps or more)

\section{Mac - Requires Mediasite 4.3 and later}

- Mac OS X 10.4.8 or later operating system

- Safari 2.0.4 or later or Firefox 2.0 or later Web browser

- Microsoft Silverlight 1.0 or later (viewers are prompted to install this plug-in when attempting to view a presentation).

- Broadband internet connection (256 Kbps or more)

Hospital for Special Surgery is pleased to share with you its copyright statement and privacy policy. You can find them here:

http://www.hss.edu/terms-of-use.asp\#Copyright_ and Trademark Information

Privacy_Policy

\section{Instructions for Course Evaluation and CME Credit:}

In order to earn CME credit, you must complete an online or print post test and evaluation following the completion of this activity. There is a passing requirement of $70 \%$. Once you complete the post test and subsequent evaluation, a certificate will be available for you to print.

For questions related to the post test and subsequent evaluation, please contact the HSS Office of Continuing Medical Education at professionaleducation@hss.edu or 212.606.1547.

\section{Option 1: Take the post-test on-line.}

1. Go to the HSS Journal homepage at www.springer.com/ hss.

2. Click on CME articles.

3. Click on "Periprosthetic Joint Infection in Patients with Inflammatory Joint Disease: a Review of Risk Factors and Current Approaches to Diagnosis and Management" to view the full-text pdf article.

4. After you have reviewed the article click on 'Complete the current CME Test Online' to register and complete the test.

Option 2: Take the post-test via hard copy printed in the Journal.

You may complete the hard copy of the post test. Please mail the test to:

Journal CME Post-Test

Office of Continuing Medical Education

Education \& Academic Affairs

535 East $70^{\text {th }}$ Street

New York, NY 10021 


\title{
Periprosthetic Joint Infection in Patients with Inflammatory Joint Disease: a Review of Risk Factors and Current Approaches to Diagnosis and Management
}

\author{
Todd A. Morrison, BS • Mark Figgie, MD • Andy O. Miller, MD • Susan M. Goodman, MD
}

Received: 22 November 2012/Accepted: 9 April 2013 /Published online: 3 July 2013

(C) Hospital for Special Surgery 2013

\begin{abstract}
Background: Prevention, early identification, and effective management of periprosthetic joint infection (PJI) in patients with inflammatory joint disease (IJD) present unique challenges for physicians. Discontinuing disease-modifying anti-rheumatoid drugs (DMARDs) perioperatively may reduce immunosuppression and infection risk at the expense of increasing disease flares. Interpreting traditional diagnostic markers of PJI can be difficult due to disease-related inflammation. Purposes: This review is designed to answer how to (1) manage immunosuppressive/DMARD therapy perioperatively, (2) diagnose PJI in patients with IJD, and (3)
\end{abstract}

$\overline{\text { Electronic supplementary material The online version of this article }}$ (doi:10.1007/s11420-013-9338-8) contains supplementary material, which is available to authorized users.

T. A. Morrison, BS $(\bowtie)$

Jefferson Medical College,

1025 Walnut Street,

Philadelphia, PA 19107, USA

e-mail: toddamorrison@gmail.com

M. Figgie, MD

Department of Orthopedic Surgery,

Hospital for Special Surgery,

535 East 70th Street,

New York, NY 10021, USA

A. O. Miller, MD

Division of Infectious Diseases,

Hospital for Special Surgery,

535 East 70th Street,

New York, NY 10021, USA

S. M. Goodman, MD

Department of Rheumatology,

Hospital for Special Surgery,

535 East 70th Street,

New York, NY 10021, USA treat PJI in this population. Methods: The PubMed database was searched for relevant articles with subsequent review by independent authors. Results: While there is evidence to support the use of methotrexate perioperatively in RA patients, it remains unclear whether using anti-tumor necrosis factor medications perioperatively increases the risk of surgical site infections. Serum erythrocyte sedimentation rate and C-reactive protein can be useful for diagnosis of PJI in this population, but only as part of comprehensive workup that ultimately relies upon sampling of joint fluid. Management of PJI depends on several clinical factors including duration of infection and the likelihood of biofilm presence, the infecting organism, sensitivity to antibiotic therapy, and host immune status. The evidence suggests that two-stage revision or resection arthroplasty is more likely to eradicate infection, particularly when MRSA is the pathogen. Conclusion: Immunosuppression and baseline inflammatory changes in the IJD population can complicate the prevention, diagnosis, and treatment of PJI. Understanding the increase in risk associated with IJD and its treatment is essential for proper management when patients undergo lower extremity arthroplasty.

Keywords periprosthetic joint infection.

inflammatory arthritis · total joint arthroplasty

\section{Introduction}

Periprosthetic joint infection (PJI) is a serious complication of total joint arthroplasty (TJA). The incidence of PJI is increasing along with the rise in the incidence and prevalence of total joint arthroplasties. According to an analysis of Nationwide Inpatient Sample data from 2001 to 2010, the incidence of infection following total knee (TKA) and total hip arthroplasty was 2.4 and $2.0 \%$, respectively. During this time, the annual cost of revisions for infection increased from $\$ 320$ million to $\$ 566$ million and is projected to exceed $\$ 1.62$ billion by 2020 [19]. 
Of particular concern is a subgroup of patients with inflammatory joint disease (IJD). This group includes patients with rheumatoid arthritis (RA), juvenile inflammatory arthritis, and spondylarthritis such as ankylosing spondylitis and psoriatic arthritis (PsA). It has been shown that patients with RA are at a 1.6 times greater risk of revision TKA for PJI with a 5-year survivorship rate of 98.9 versus $99.3 \%$ for patients with osteoarthritis (OA) [29]. Sources for PJI in all patients include hematogenous seeding and contiguous (usually primary surgical site) infection. Contiguous infection is likely due to skin flora and typically occurs within 3 months after arthroplasty, whereas hematogenous seeding can be from a variety of pathogens, often from an abnormal mucosal (oral, GI) or cutaneous site. While Staphylococcus epidermis is the leading pathogen in all PJI, a report of 200 PJI cases in RA patients identified Staphylococcus aureus as the most common pathogen [3]. A recent comparison of PJI cases caused by either contiguous or hematogenous $S$. aureus infection found that PJI in patients with RA were equally likely to occur via either route [31]. Early case series of patients with PsA reported deep infection rates of 5.5-16.6\% [21, 34]. High levels of bacteria reside in psoriatic plaques, including streptococcal and staphylococcal species, which may be the source of the increase in PJI infection [11].

Once within the joint space, bacteria attach to implants via adherens and multiply to reach a quorum or predetermined concentration at which they can develop a polysaccharide matrix. This biofilm matrix creates a sophisticated microenvironment that allows bacteria to interact with one another via nanowires and shields them from antibiotics. Biofilms can increase minimal bactericidal concentrations of antibiotics by factors of more than a thousand, to concentrations that are not clinically safe or achievable. Biofilms require variable amounts of time (days to weeks) to grow and become fully established, and it is therefore unsurprising that early diagnosis and treatment of PJI has been associated with improved success of hardware-retention strategies [35].

Inflammatory joint disease has been identified as an independent risk factor for PJI. Bongartz et al. found an overall infection rate of $3.7 \%$ in RA patients, with $5.9 \%$ of revision arthroplasties complicated by PJI and 30.4\% of all infections occurring in joints that had been previously affected by PJI. Both revision arthroplasty and prior PJI were identified as risk factors for PJI. In this RA population, risk of PJI was only $3.5 \%$ if revision arthroplasty surgery was performed in a joint with no prior PJI [5]. Active RA is also a risk factor for infection, and patents with more swollen and tender joints are at higher risk for infection, adding to the conflict between holding and continuing disease-modifying anti-rheumatoid drugs (DMARDs) perioperatively [1].

Given the need for immunosuppression and baseline inflammatory characteristics of patients with IJD, preventing, diagnosing, and treating PJI presents several challenges. The objective of this literature review is to synthesize data in order to optimize the management of perioperative DMARD therapy, the diagnosis of PJI, and treatment of PJI in this challenging population.

\section{Methods}

To answer the questions proposed in this review, the PubMed database was searched for English language publications through September 2012 using the terms "inflammatory arthritis," "arthroplasty," and "infection." The initial search returned a total of 1,477 publications of which 1,178 were full-text articles. These articles were then searched for the keywords "DMARD," "diagnosis," and "treatment." The titles and abstracts of the resulting articles were then screened for relevance. Relevant articles included randomized control trials as well as prospective cohort and retrospective studies. A total of 33 relevant articles were identified and were reviewed by the authors.

\section{Results}

The relevant literature on how perioperative immunosuppressive DMARD therapy should be managed is limited to the RA population. Grennan et al. examined the perioperative use of methotrexate in RA patients and found no difference in early or late infection rates between those patients who continued or discontinued drug therapy 4 weeks prior to surgery $[15,33]$. Their initial study demonstrated an increase in disease flares in those patients who stopped methotrexate, leading to the recommendation that this DMARD be continued perioperatively. A 2006 literature review on perioperative DMARDs noted this finding but was unable to make definitive recommendations on the perioperative use of corticosteroids or other non-biologic DMARDs due to insufficient prospective data [27]. There is also a paucity of high-quality data regarding the perioperative use of biologic DMARDs including anti-tumor necrosis factor (anti-TNF) drugs and interleukin-6 antagonists. A recent retrospective review demonstrated an acute surgical site infection rate of $0.7 \%$ in RA patients and identified the use of anti-TNF DMARDs like infliximab or etanercept and longer RA duration as significant risk factors for infection overall [23]. However, anti-TNF therapy was not an associated risk factor for deep surgical site infections. There was no information regarding the interruption of anti-TNF therapy as it relates to rheumatoid flares. The perioperative use of anti-TNF medications in RA patients undergoing lower extremity TJA has been examined in three observational studies which all demonstrated conflicting results. A previous review determined that only one contained high-quality data, albeit with low statistical power [8].

Several diagnostic tests for PJI in patients with IJD have been examined. C-reactive protein (CRP) $>1 \mathrm{mg} / \mathrm{dL}$ and erythrocyte sedimentation rate $(E S R)>30 \mathrm{~mm} / \mathrm{h}$ at a minimum of 3 weeks postoperatively were found to have inconsistent sensitivities and specificities for the diagnosis of hip and knee PJI in several studies (Table 1). While patients with connective tissue disorders were included in these studies, they were not analyzed independently from osteoarthritis. One retrospective review of 228 suspected PJIs found that CRP was more sensitive than ESR when infection was defined by a minimum of one positive intraoperative culture for virulent organisms such as streptococci species or Gram-negative bacterium or three positive intraoperative cultures for less virulent organisms like coagulase-negative staphylococci or propionibacterium species [4]. 
Table 1 Summary of previous studies examining erythrocyte sedimentation rate (ESR), and C-reactive protein (CRP) for the diagnosis of periprosthetic joint infection

\begin{tabular}{|c|c|c|c|c|}
\hline Article & Joint & ESR threshold $(\mathrm{mm} / \mathrm{h})$ & CRP threshold (mg/L) & Management of IJD patients \\
\hline Spangehl et al. [32] & Hip & $30(\mathrm{SN}=82 \%, \mathrm{SP}=85 \%)$ & $10(\mathrm{SN}=96 \%, \mathrm{SP}=92 \%)$ & $\begin{array}{l}23 \text { patients with IJD were excluded } \\
\text { from analysis }\end{array}$ \\
\hline Baré et al. [2] & Knee & $30(\mathrm{SN}=63 \%, \mathrm{SP}=55 \%)$ & $10(\mathrm{SN}=60 \%, \mathrm{SP}=63 \%)$ & $\begin{array}{l}\text { No mention of how IJD patients } \\
\text { were managed during analysis }\end{array}$ \\
\hline Bernard et al. [4] & Hip \& Knee & $30(\mathrm{SN}=87 \%, \mathrm{SP}=47 \%)$ & $10(\mathrm{SN}=97 \%, \mathrm{SP}=81 \%)$ & Included in analysis \\
\hline Della Valle et al. [10] & Knee & $30(\mathrm{SN}=90.2 \%, \mathrm{SP}=66 \%)$ & $10(\mathrm{SN}=95.1 \%, \mathrm{SP}=75.5 \%)$ & Excluded \\
\hline Greidanus et al. [14] & Knee & $22.5(\mathrm{SN}=93 \%, \mathrm{SP}=83 \%)$ & $13.5(\mathrm{SN}=91 \%, \mathrm{SP}=86 \%)$ & Excluded \\
\hline Schinsky et al. [28] & Hip & $30(\mathrm{SN}=97 \%, \mathrm{SP}=39 \%)$ & $10(\mathrm{SN}=94 \%, \mathrm{SP}=71 \%)$ & Excluded \\
\hline Cipriano et al. [7] & Hip \& Knee & $\begin{array}{l}30 \text { in } \operatorname{IJD}(\mathrm{SN}=94.4 \% \\
\mathrm{SP}=59.9 \%) \\
32 \text { in } \mathrm{OA}(\mathrm{SN}=87.2 \% \\
\mathrm{SP}=67.1 \%)\end{array}$ & $\begin{array}{l}17 \text { in } \operatorname{IJD}(\mathrm{SN}=93.8 \%, \\
\mathrm{SP}=70.3 \%) \\
15 \text { in } \mathrm{OA}(\mathrm{SN}=85.8 \% \\
\mathrm{SP}=83.4 \%)\end{array}$ & $\begin{array}{l}\text { Separate and comparative analyses } \\
\text { of } 61 \text { IJD patients and } 810 \\
\text { OA patients }\end{array}$ \\
\hline
\end{tabular}

$I J D$ inflammatory joint disease, $S N$ sensitivity, $S P$ specificity

Although other studies $[10,14,28]$ have determined optimal cutoff values for serum ESR and CRP diagnostic for infection, patients with inflammatory arthritis were excluded from the analysis. Despite this, these studies demonstrated the strong sensitivity and specificity of serum ESR and CRP in the detection of PJI. To date, only one study has specifically examined inflammatory markers for PJI in patients with IJD. Cipriano et al. recently compared the optimal diagnostic threshold of ESR and CRP values for 61 patients with inflammatory arthritis and 810 patients with noninflammatory arthritis with suspected chronic PJI and found similar cutoff values for each group. Furthermore, serum ESR and CRP had comparable sensitivities, specificities, negative predictive values, and positive predictive values for PJI in both inflammatory and noninflammatory arthritis [7]. As expected, they did observe a significantly higher incidence of PJI in patients with IJD. Several synovial fluid markers have also been investigated. Cipriano et al. observed that optimal cutoff values for WBC count and polymorphic neutrophil percentage (PMN\%) do not differ in patients with underlying inflammatory conditions and that synovial fluid WBC count and differential had the highest sensitivity and specificity for differentiating septic from aseptic failure in patients with inflammatory and noninflammatory arthritis [7] (see Table 2). However, inflammatory synovitis may similarly elevate these parameters. Given this overlap in values, further evaluation for infection in patients with inflammatory arthritis and elevated WBC count and $\mathrm{PMN} \%$ in the appropriate clinical setting is recommended.

There are limited data regarding the optimal treatment of PJI in patients with IJD. A recent retrospective review of revision TKA in patients with RA describes the outcomes of 18 cases of subacute and chronic PJI [12]. Of the 12 patients who received a two-stage revision, five of them subsequently died within 6 months, while three went on to have failures secondary to reinfection at a mean of 39.6 months. Despite these failures, the mean postoperative Knee Society Score, function score, and range of motion significantly improved postoperatively for all patients who had revision for infection. The authors could not report implant survival probability for revision secondary to infection due to the relatively few cases; however, implant survival for both mechanical failure and infection was $76.8 \%$ at 59 months. It is difficult to make any

Table 2 Summary of previous studies examining synovial white blood cell (WBC) count and differential (PMN\%) for the diagnosis of periprosthetic joint infection

\begin{tabular}{|c|c|c|c|c|}
\hline Article & Joint & $\begin{array}{l}\text { Synovial WBC Count } \\
\text { Threshold }(\mathrm{WBC} / \mu \mathrm{L})\end{array}$ & PMN\% Threshold & Management of IJD Patients \\
\hline Spangehl et al. [32] & Hip & $1,100(\mathrm{SN}=20 \%, \mathrm{SP}=96 \%)$ & $75 \%(\mathrm{SN}=24 \%, \mathrm{SP}=89 \%)$ & $\begin{array}{l}23 \text { patients with IJD were excluded from } \\
\text { analysis }\end{array}$ \\
\hline Kersey et al. [17] & Knee & 2,000 & $50 \%$ & $\begin{array}{l}>50 \% \text { PMN seen only in four patients } \\
\text { with RA with high rate of false positive }\end{array}$ \\
\hline Mason et al. [20] & Knee & $2,500(\mathrm{SN}=69 \%, \mathrm{SP}=98 \%)$ & $60 \%(\mathrm{SN}=76 \%, \mathrm{SP}=89 \%)$ & $\begin{array}{l}\text { Analysis included four patients with IJD, } \\
\text { however no conclusions were made }\end{array}$ \\
\hline Trampuz et al. [36] & Knee & $1,700(\mathrm{SN}=94 \%, \mathrm{SP}=88 \%)$ & $65 \%(\mathrm{SN}=97 \%, \mathrm{SP}=98 \%)$ & Excluded \\
\hline Della Valle et al. [4] & Knee & $3,000(\mathrm{SN}=100 \%, \mathrm{SP}=98.1 \%)$ & $\begin{array}{l}65 \%(\mathrm{SN}=97.6 \% \\
\mathrm{SP}=84.9 \%)\end{array}$ & Excluded \\
\hline Schinsky et al. [28] & Hip & $4,200(\mathrm{SN}=84 \%, \mathrm{SP}=93 \%)$ & $80 \%(\mathrm{SN}=84 \%, \mathrm{SP}=82 \%)$ & Excluded \\
\hline Cipriano et al. [7] & $\begin{array}{l}\text { Hip \& } \\
\text { Knee }\end{array}$ & $\begin{array}{l}3,444 \text { in } \mathrm{IJD}(\mathrm{SN}=88.2 \% \\
\mathrm{SP}=80.0 \%) \\
3450 \text { in } \mathrm{OA}(\mathrm{SN}=91.0 \% \\
\mathrm{SP}=93.0 \%)\end{array}$ & $\begin{array}{l}75 \% \text { in } \mathrm{IJD}(\mathrm{SN}=100 \% \\
\mathrm{SP}=81.8 \%) \\
78 \% \text { in } \mathrm{OA}(\mathrm{SN}=95.5 \% \\
\mathrm{SP}=87.3 \%)\end{array}$ & $\begin{array}{l}\text { Separate and comparative analyses of } \\
61 \text { IJD patients and } 810 \text { OA patients }\end{array}$ \\
\hline
\end{tabular}


conclusions about revision TKA for PJI in IJD patients based on these data alone. A larger case series of $200 \mathrm{RA}$ patients with PJI found rates of 5-year survival, defined as time free of treatment failure, for patients with debridement and retention of components, two-stage revision, and resection arthroplasty to be 32,79 , and $62 \%$, respectively [3]. Patients with younger prosthesis age and shorter duration of symptoms were more likely to be treated with debridement and retention of components, while older patients with $S$. aureus infection were more likely to be treated with resection arthroplasty without implantation. Debridement and retention of components had 5.9 times increased risk of treatment failure when compared to two-stage revision. The success of two-stage revision and resection arthroplasty in this case series may be the result of prosthesis removal, whereas debridement and retention may provide a continued source of reinfection due to biofilm formation.

\section{Discussion}

Prevention, early identification, and effective management of PJI in the IJD population present unique challenges for physicians. Discontinuing DMARDs perioperatively may reduce immunosuppression at the expense of increasing disease flares. Interpreting traditional diagnostic markers of PJI can be complicated by baseline disease-related inflammation. Eradicating infection can be difficult. The purpose of this review was to answer the following clinical questions: what is the optimal perioperative management of immunosuppressive DMARD therapy, how is PJI diagnosed in this population, and how should PJI be treated in this population?

One of the major limitations of this review is that patients with IJD undergoing lower extremity TJA are inconsistently categorized by diagnosis. For instance, several of the articles classified multiple types of arthritis under a single category of "inflammatory arthritis," while other articles were specific in regard to diagnosis such as RA or PsA, making it difficult to compare and contrast the findings of multiple studies. Despite this, it was possible to enumerate and discuss the major questions of this review using the available literature.

While there is evidence to support the use of methotrexate perioperatively, it remains unclear whether patients with RA using anti-TNF medications perioperatively are at increased risk of surgical site infections compared to patients on synthetic DMARDs or corticosteroids. However, given the devastating consequences of PJI, the American College of Rheumatology recommends that biologic DMARDs should not be used at least 1 week prior to surgery, with the possibility of an earlier preoperative cessation as determined by pharmacokinetic half-life, and restarted 2 weeks postoperatively [26], once the incision is healed. Use of biologic agents in the perioperative period may have additional consequences caused by masking the normal inflammatory febrile response. Patients on tocilizumab, a humanized anti-IL-6 receptor antibody, may not mount an appropriate inflammatory response to stress or infection and fail to mount the normal rise in CRP and fever. This may impact wound healing and may make recognition of acute perioperative events more difficult $[16,22]$.
Serum ESR and CRP can be useful for diagnosis of PJI in this population, but only as part of comprehensive workup that ultimately relies upon sampling of joint fluid. Neither serum ESR or CRP alone is adequate to diagnose or exclude infection, as they vary with disease activity in patients with inflammatory arthritis. C-reactive protein has been shown to have the highest correlation with disease activity in patients with rheumatoid arthritis [38] and ankylosing spondylitis [37]. Additional variability in ESR and CRP levels is seen in early- versus late-onset RA, although these differences disappear after age adjustment [25]. Therefore, it is important to keep in mind the natural variability of these parameters. Inflammatory synovitis may similarly elevate these markers and should be considered in cases where no evidence of infection is apparent on culture or histological examination of periprosthetic tissue. While joint aspirate and intraoperative culture have traditionally served as the gold standard for diagnosis of PJI, a multicenter study found a relatively high percentage of both falsenegative and false-positive cases, regardless of whether or not perioperative antibiotics were received [6]. The study did not assess the utility of microbial culture results in optimizing the antibiotic treatment of PJI. Furthermore, this study excluded patients with inflammatory arthritis and underscores the importance of synovial fluid culture in the IJD population due to the variability in values like ESR and CRP and synovial fluid cell counts for patients with IJD [7]. Therefore, the value of synovial culture may outweigh the risks of false-positives. Interpreting the sometimes-conflicting results of multiple diagnostic tests presents a challenge for establishing a definitive diagnosis, regardless of whether a patient has IJD. The Musculoskeletal Infection Society states that an infection exists if any of the following are present: (1) sinus tract communicates with prosthesis or (2) a pathogen is isolated by cultures from two separate tissue samples, or (3) four of six criteria are met including elevated serum ESR and CRP, elevated synovial WBC count, elevated synovial PMN\%, purulence in the affected joint, pathogen isolated by culture from single tissue sample, or the presence of greater than five neutrophils per high-powered field at 400 times magnification in periprosthetic tissue [24]. This consensus does not differentiate criteria for patients with IJD. However, the AAOS has suggested guidelines for the diagnosis of periprosthetic knee infection in a high-risk population that includes immunosuppressed patients [9] (see Fig. 1).

Management of PJI depends on several clinical factors including the likelihood of biofilm presence, the infecting organism, sensitivity to antibiotic therapy, and host immune status. The evidence suggests that two-stage revision or resection arthroplasty is more likely to eradicate infection, particularly when MRSA is the pathogen. PJI has been divided into four categories based on clinical presentation of infection and its temporal relationship to the index surgery [30]. This treatment algorithm was evaluated in 114 periprosthetic knee infections, and an overall infection control rate of $100 \%$ at a minimum of 2 years was seen with $82 \%$ of the first-line treatments being successful [18]. Although this study did not include patients with IJD, conservative management with a two-stage revision showed a higher cure rate than any other technique in 


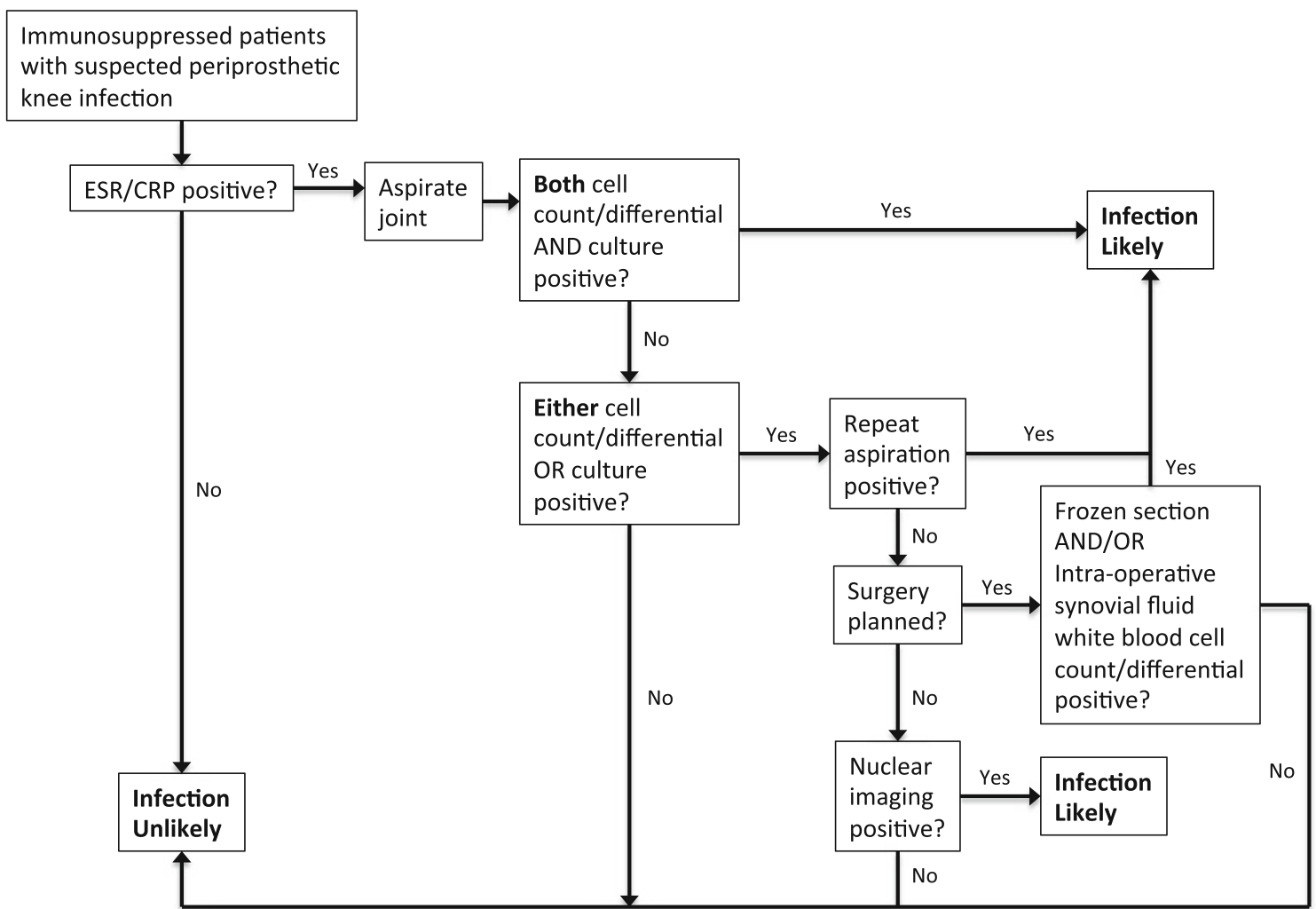

Fig. 1. The American Academy of Orthopaedic Surgeons' (AAOS) algorithm for diagnosing periprosthetic knee infection in a high-risk population that includes immunosuppressed patients. The following states are considered indicative of immunosuppression: suppressive medication such as prednisone, infliximab, adalimumab, methotrexate and etanercept, autoimmune diseases (lupus, rheumatoid arthritis, ankylosing spondylitis, Reiter's syndrome, psoriatic arthropathy), and inflammatory arthritis. ESR erythrocyte sedimentation rate, CRP C-reactive protein. Modified with permission from American Academy of Orthopaedic Surgeons: (Clinical Practice Guideline on The Diagnosis of Periprosthetic Infections of the Hip and Knee.) Rosemont, IL, American Academy of Orthopaedic Surgeons, June 2010. http://www.aaos.org/Research/guidelines/PJIguideline.pdf

osteoarthritis patients. In certain clinical situations such as in early/acute infections, or when patients have major comorbidities making them poor operative candidates, retention of hardware can be selected. However, it has a lower rate of cure and patients usually require continued infection control and suppression via prolonged oral antibiotics [13].

In conclusion, immunosuppression and baseline inflammation in the IJD population can complicate the prevention, diagnosis, and treatment of PJI. Understanding the current literature regarding this topic is essential for proper management of patients undergoing lower extremity arthroplasty.

\section{Disclosures}

Conflict of Interest: Todd A. Morrison, BS, Andy O. Miller, MD, and Susan M. Goodman, MD have declared that they have no conflict of interest. Mark Figgie, MD received research grants from Ethicon and Baxter and own stocks in Mekanika, outside the work.

Human/Animal Rights: All procedures followed were in accordance with the ethical standards of the responsible committee on human experimentation (institutional and national) and with the Helsinki Declaration of 1975, as revised in 2008 (5).

Human/Animal Rights: This article does not contain any studies with human or animal subjects performed by any of the authors.

Informed Consent: N/A
Required Author Forms Disclosure forms provided by the authors are available with the online version of this article.

\section{References}

1. Au K, Reed G, Curtis J, et al. High disease activity is associated with an increased risk of infection in patients with rheumatoid arthritis. Ann Rheum Dis. 2011;70:785-791.

2. Baré J, Macdonald S, Bourne R. Preoperative Evaluations in Revision Total Knee Arthroplasty. Clin Orthop. 2006;446:40-44.

3. Berbari EF, Osmon D, Duffy MC, et al. Outcome of prosthetic joint infection in patients with rheumatoid arthritis: the impact of medical and surgical therapy in 200 episodes. Clin Infect Dis. 2006;42:216-223.

4. Bernard L, Lübbeke A, Stern R, et al. Value of peroperative investigation in diagnosing prosthetic joint infection: retrospective cohort study and literature review. Scan J Infect Dis. 2004;36:410-416.

5. Bongartz $\mathrm{T}$, Halligan $\mathrm{C}$, Osmon $\mathrm{D}$, et al. Incidence and risk factors of prosthetic joint infection after total hip or knee replacement in patients with rheumatoid arthritis. Arthritis Rheum. 2008;59:1713-1720.

6. Breidahl WH, Newman JS, Taljanovic MS, Adler RS. Power Doppler sonography in the assessment of musculoskeletal fluid collection. Am J Roentgenol. 1996;166(6):1444-1446.

7. Cipriano CA, Brown NM, Michael AM, Moric M, Sporer SM, Della VC. Serum and synovial fluid analysis for diagnosing chronic periprosthetic infection in patients with inflammatory arthritis. $J$ Bone Joint Surg Am. 2012;94:594-600. 
8. Da Cunha B, Maria Henrique Da Mota L, Dos Santos-Neto L. Risk of orthopedic surgical site infections in patients with rheumatoid arthritis treated with antitumor necrosis factor alpha therapy. Int J Rheumatol. 2012;2012:1-5.

9. Della Valle C, Parvizi J, Bauer TW, et al. American Academy of Orthopaedic Surgeons clinical practice guideline on: the diagnosis of periprosthetic joint infections of the hip and knee. J Bone Joint Surg Am. 2011;93:1355-1357.

10. Della Valle CJ, Sporer SM, Jacobs JJ, Berger RA, Rosenberg AG, Paprosky WG. Preoperative testing for sepsis before revision total knee arthroplasty. J Arthroplasty. 2007;22:90-93.

11. Fahlén A, Engstrand L, Baker BS, Powles A, Fry L. Comparison of bacterial microviota in skin biopsies from normal and psoriatic skin. Arch Dermatol Res. 2012;304(1):15-22.

12. Garcia R, Hardy B, Kraay M, Goldberg V. Revision total knee arthroplasty for aseptic and septic causes in patients with rheumatoid arthritis. Clin Orthop. 2010;468:82-89.

13. Gardner J, Gioe TJ, Tatman P. Can this prosthesis be saved? Implant salvage attempts in infected primary TKA. Clin Orthop. 2011;469(4):970-976.

14. Greidanus N, Masri B, Garbuz D, et al. Use of erythrocyte sedimentation rate and C-reactive protein level to diagnose infection before revision total knee arthroplasty. A prospective evaluation. $J$ Bone Joint Surg. 2007;89:1409-1416.

15. Grennan DM, Gray J, Loudon J, Fear S. Methotrexate and early postoperative complications in patients with rheumatoid arthritis undergoing elective orthopaedic surgery. Ann Rheum Dis. 2001;60:214-217.

16. Hirao M, Hashimoto J, Tsuboi $\mathrm{H}$, et al. Laboratory and febrile features after joint surgery in patients with rheumatoid arthritis treated with tocilizumab. Ann Rheum Dis. 2009;68:654-657.

17. Kersey R, Benjamin J, Marson B. White blood cell counts and differential in synovial fluid of aseptically failed total knee arthroplasty. J Arthroplasty. 2000;15:301-304.

18. Kim Y, Choi Y, Kim J. Treatment based on the type of infected TKA improves infection control. Clin Orthop. 2011;469:977-984.

19. Kurtz SM, Lau E, Watson H, Schmier JK, Parvizi J. Economic burden of periprosthetic joint infection in the United States. J Arthroplasty. 2012; 27: 61-5.e1

20. Mason JB, Fehring T, Odum S, Griffin WL, Nussman DS. The value of white blood cell counts before revision total knee arthroplasty. J Arthroplasty. 2003;18:1038-1043.

21. Menon TJ, Wroblewski BM. Charnley low-friction arthroplasty in patients with psoriasis. Cli Orthop Relat Res. 1983;176:127-128.

22. Momohara S, Hashimoto J, Tsuboi H et al. Analysis of perioperative clinical features and complications after orthopaedic surgery in rheumatoid arthritis patients treated with tocilizumab in a realworld setting: results from the multicentre TOcilizumab in Perioperative Period (TOPP) study. Mod Rheumatol. 2012; doi: $10.1007 / \mathrm{s} 10165-012-0683-0$

23. Momohara S, Kawakami K, Iwamoto T, et al. Prosthetic joint infection after total hip or knee arthroplasty in rheumatoid arthritis patients treated with nonbiologic and biologic disease-modifying antirheumatic drugs. Mod Rheumatol. 2011;21:469-475.

24. Musculoskeletal Infection Society Workgroup. New definition for periprosthetic joint infection. J Arthroplasty. 2011;26:1136-1138.
25. Ranganath VK, Elashoff DA, Khanna D, et al. Age adjustment corrects for apparent differences in erythrocyte sedimentation rate and C-reactive protein values at the onset of seropositive rheumatoid arthritis in younger and older patients. $J$ Rheumatol. 2005;32:1040-1042.

26. Saag K, Teng G, Patkar N, et al. American College of Rheumatology 2008 recommendations for the use of nonbiologic and biologic disease-modifying antirheumatic drugs in rheumatoid arthritis. Arthritis Rheum. 2008;59:762-784.

27. Scanzello C, Figgie M, Nestor B, Goodman S. Perioperative management of medications used in the treatment of rheumatoid arthritis. HSS J. 2006;2:141-147.

28. Schinsky M, Della Valle C, Sporer S, Paprosky W. Perioperative testing for joint infection in patients undergoing revision total hip arthroplasty. J Bone Joint Surg. 2008;90:1869-1875.

29. Schrama J, Espehaug B, Hallan G, et al. Risk of revision for infection in primary total hip and knee arthroplasty in patients with rheumatoid arthritis compared with osteoarthritis: a prospective, population-based study on 108,786 hip and knee joint arthroplasties from the Norwegian Arthroplasty Register. Arthritis Care Res. 2010;62:473-479.

30. Segawa H, Tsukayama DT, Kyle RF, Becker DA, Gustilo RB. Infection after total knee arthroplasty. A retrospective study of the treatment of eighty-one infections. J Bone Joint Surg Am. 1999;81:1434-1445.

31. Sendi P, Banderet F, Graber P, Zimmerli W. Clinical comparison between exogenous and haematogenous periprosthetic joint infections caused by Staphylococcus aureus. Clin Microbiol Infect. 2011;17:1098-1100.

32. Spangehl MJ, Masri BA, O'Connell JX, Duncan CP. Prospective analysis of preoperative and intraoperative investigations for the diagnosis of infection at the sites of two hundred and two revision total hip arthroplasties. J Bone Joint Surg Am. 1999;81:672-683.

33. Sreekumar R, Gray J, Kay P, Grennan DM. Methotrexate and post operative complications in patients with rheumatoid arthritis undergoing elective orthopaedic surgery - a ten year follow-up. Acta Orthop Belg. 2011;77:823-826.

34. Stern SH, Insall JN, Windsor RE, Inglis AE, Dines DM. Total knee arthroplasty in patients with psoriasis. Clin Orthop Relat Res. 1989;248:108-110

35. Tattevin P, Crémieux AC, Pottier P, Huten D, Carbon C. Prosthetic joint infection: when can prosthesis salvage be considered? Clin Infect Dis. 1999;29:292-295.

36. Trampuz A, Hanssen A, Osmon D, Mandrekar J, Steckelberg JM, Patel R. Synovial fluid leukocyte count and differential for the diagnosis of prosthetic knee infection. Am J Med. 2004;117:556562

37. Yildirim K, Erdal A, Karatay S, Melikoğlu MA, Uğur M, Senel K. Relationship between some acute phase reactants and the Bath Ankylosing Spondylitis Disease Activity Index in patients with ankylosing spondylitis. South Med J. 2004;97:350-353.

38. Yildirim K, Karatay S, Melikoglu MA, Gureser G, Ugur M, Senel $\mathrm{K}$. Associations between acute phase reactant levels and disease activity score (DAS28) in patients with rheumatoid arthritis. Ann Clin Lab Sci. 2005;34:423-426. 


\title{
Periprosthetic Joint Infection in Patients with Inflammatory Joint Disease: a Review of Risk Factors and Current Approaches to Diagnosis and Management
}

\author{
Todd A. Morrison, BS • Mark Figgie, MD • Andy O. Miller, MD • Susan M. Goodman, MD
}

Received: 22 November 2012/Accepted: 9 April 2013 /Published online: 3 July 2013

(C) Hospital for Special Surgery 2013

\section{CME Questions}

1) Patients with Rheumatoid arthritis are at higher risk than patients with osteoarthritis for prosthetic joint infection.
a) True
b) False

2) Risk factors for prosthetic joint infection include:
a) rheumatoid arthritis
b) revision arthroplasty
c) prior prosthetic joint infection
d) active RA
e) all of the above
f) none of the above

3) Prior to arthroplasty, methotrexate treatment for RA should be:
a) held for 2 weeks
b) dose should be increased
c) usual dose should be continued

4) Prosthetic joint infection is more likely to be eradicated with:

a) debridement and retention of the prosthesis plus antibiotic therapy

b) oral antibiotics given over a prolonged period

c) 2-stage explant, debridement, and re-implant after antibiotic therapy

5) Formation of a bacterial biofilm:
a) occurs within 24 hours of prosthetic joint infection
b) creates a sophisticated matrix which shields bacteria from antibiotics
c) results in in lower bactericidal concentrations

6) Which of the following factors is/are associated with resection arthroplasty without reimplantation in rheumatoid arthritis patients with periprosthetic knee infection?
a) increased age
b) S. aureus pathogen
c) prior PJI
d) a and $b$
e) all of the above

7) A diagnosis periprosthetic joint infection can be made if:

a) a sinus tract communicates with prosthesis

b) a pathogen is isolated by cultures from two separate tissue samples

c) four of six criteria are met including elevated serum ESR and CRP, elevated synovial WBC count, elevated synovial PMN\%, purulence in the affected joint, pathogen isolated by culture from single tissue sample, or the presence of greater than five neutrophils per high-powered field at 400 times magnification in periprosthetic tissue

d) all of the above

8) There is definitive evidence to support withdrawling biological DMARDs like anti-TNF inhibitors prior to lower extremity arthroplasty.
a) True
b) False

9) Elevated serum ESR and CRP alone are enough to diagnose PJI in patients with IJD.
a) True
b) False 


\section{CME Registration Form}

The Accreditation Council for Continuing Medical Education requires that accredited CME providers evaluate and assess the effectiveness of all Continuing Medical Education accredited activities. This evaluation will help us document our efforts to address physicians' practice gaps and educational needs.

Should you have any questions, please contact the Office of Continuing Medical Education at professionaleducation@hss.edu. Thank you.

Expires June 15, 2014

\section{Please complete the following contact information:}

First Name:

Last Name:

Title (i.e. $\mathrm{MD}, \mathrm{PhD}, \mathrm{NP}$ )

Organization

Street Address

City, State, Zip

Country

Email Address

Please indicate your profession:
- Family Medicine
○ Orthopaedic Surgeons
○ Fellows
Residents
- General Medicine
○ Rheumatologist
- Medical Students
o Other

Please give us your feedback by completing this evaluation. All fields are required.

\section{How certain are you to implement the following strategies in your practice:}

(Scale: $1=$ Not At All Certain; 7= Very Certain)

Recognize the signs and symptoms of PJI, such as an increase in pain and swelling, drainage from the joint, or systemic features such as fever or fatigue, in patients with inflammatory arthritis.

Be able to describe and safely prescribe DMARDs, such as methotrexate and plaquenil, to avoid increased risk of infection and achieve optimal outcomes.

Be able to implement additional work-up that may be necessary if certain inflammatory markers, such as ESR and CRP levels, are elevated in patients with PJI who have inflammatory arthritis.

Understand the treatment options and the risks and benefits of two-stage revision or resection arthroplasty, compared to other therapies when PJI is diagnosed in patients with inflammatory arthritis. 


\section{Enhanced practitioner}

(Scale: $1=$ Not At All Certain; $7=$ Very Certain)

Enhanced practitioner

How certain are you that your practice will be enhanced by participating in this course?

Please respond to the following statement:

As a result of what I have learned, I will make the following change(s) in my practice:

1.

2.

3.

Overall, the content covered in this journal article has been useful and relevant to my scope of practice.

$\circ$ Strongly Agree $\quad$ Agree $\circ$ Strongly Disagree $\circ$ Disagree

If you disagree, please explain why

Do you feel this activity was free of commercial bias?

$\circ$ Yes $\circ$ No

If no, please comment

Please provide at least one topic to be covered in future journal articles that would help you in your practice and/ or improve the health of your patients:

Please provide at least one suggestion for improving this journal article so that it will be more effective:

Please respond to the following statement:

Using this technology was easy.

$\circ$ Strongly Agree $\circ$ Agree $\circ$ Strongly Disagree $\circ$ Disagree

If you disagree, please explain why

How likely are you to read more journal articles offered in this electronic format?

$\circ$ Very likely $\circ$ Neutral $\bigcirc \quad$ Very unlikely

If unlikely, please explain why 